\title{
CFO Dialog 23 - Financial Leadership
}

Risikomanagement in Planung \& Controlling, Offshoring versus Outsourcing und Working Capital Management waren die Themen, denen sich der CFO-Dialog 23 in Berlin am 5. und 6. November 2012 widmete.

Besonders kontrovers wurde der Vortrag von Martin Wenderoth „Better \& Beyond Budgeting - Wie man die Planung abschafft und trotzdem erfolgreich steuert" diskutiert. Wenderoth stellte vor, wie das Pharma- und Medizinbedarfsunternehmen B. Braun Melsungen seine Planung im operativen und strategischen Controlling aufgesetzt hat. Die Möglichkeit der Steuerung des Unternehmens ohne Budgetrestriktionen, der neue Planungsprozess im Vergleich zum alten sowie die Vorteile des neuen Budgetplans standen im Fokus des Vortrags und der anschließenden Diskussion.

Dr. Ulrich Hauck von der Bayer AG zeigte in seinem Vortrag Kosteneinsparungspotenziale durch die Einführung von SSCs und Outsourcing-Konzepten am Beispiel seines Unter-

\section{Klare Entscheidungen, klare Strategie- kommunikation}

\section{Vorschau auf den WHU Campus for Strategy \& Leadership am 12.4.2012 in Düsseldorf}

Mit dem Campus for Strategy \& Leadership veranstaltet die WHU - Otto Beisheim School of Management eine jährliche Konferenz, die sich speziell an Strategie-Experten und Führungskräfte aus der Unternehmenspraxis richtet.

Unter dem diesjährigen Leitthema „Schwierige Zeiten klare Entscheidungen, klare Strategiekommunikation“ diskutieren Referenten und Teilnehmer im Rahmen von Vorträgen, interaktiven World Cafés und Networking-Veranstaltungen konkret und kompakt u. a., wie Unternehmen ihre strategische Agilität erhalten können, wie schwierige Entscheidungen getroffen werden und wie Mitarbeiter zur raschen Umsetzung von Strategien motiviert werden.

Referenten sind u. a.: nehmens auf. Die Bayer AG hat in den letzten Jahren ein weltweites Shared-Services-Netzwerk aufgebaut und die konzerninternen Strukturen harmonisiert.

Für die Teilnehmer aus dem Finanzbereich bot sich neben den interessanten Workshops und Vorträgen die Möglichkeit, in exklusiven Gesprächen hochkarätige Kollegen aus dem CFO-Umfeld kennenzulernen. Doch nicht nur zum Netzwerken lud die Konferenz ein. Gerade der aktive Erfahrungsaustausch von Teilnehmern und Referenten macht die Veranstaltungsreihe so spannend.

Weitere Informationen zum nächsten CFO-Dialog am 22./23. April 2013 in Frankfurt erhalten Sie auf der Homepage http://summits.econique.com. Als Schwerpunktthemen sind u. a. Kostensenkungsmaßnahmen und Sales Performance Controlling geplant.

Quelle: Econique

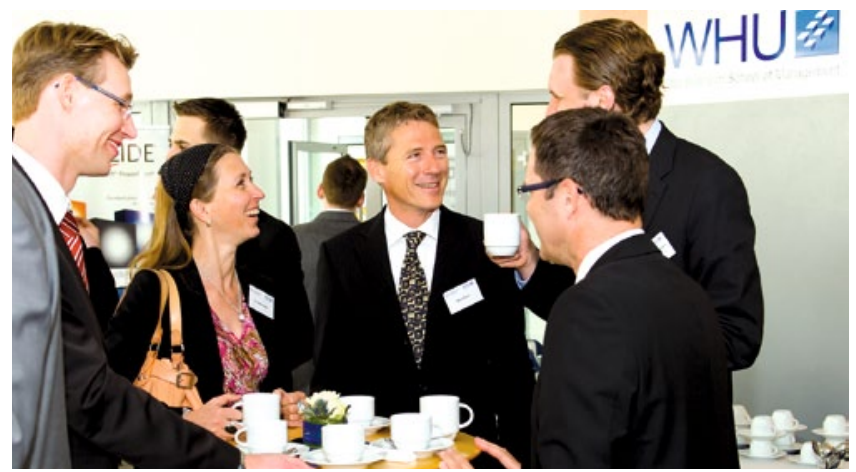

Networking beim Campus for Strategy \& Leadership

- Dr. Michael Groß, Geschäftsführer, Groß \& Cie. GmbH und dreifacher Olympiasieger

- Dr. Lars Grünert, Geschäftsführer, TRUMPF GmbH + Co. KG

- Dr. Franz-Josef Seidensticker, Director, Bain \& Company Germany

- Arndt Geiwitz, Partner, Schneider, Geiwitz \& Partner und Insolvenzverwalter u. a. bei Schlecker und Budget Autovermietung

- Dirk Metz, Staatssekretär a. D. und selbstständiger Kommunikationsberater

Weitere Informationen, ein Video mit Impressionen und Online-Anmeldung: www.strategyandleadership.de

Prof. Dr. Lutz Kaufmann 